\title{
Doxycycline in the Coronavirus Disease 2019 Therapy
}

\author{
Karolina Dorobisz (D) \\ Tadeusz Dorobisz ${ }^{2}$ \\ Dariusz Janczak ${ }^{2}$ \\ Tomasz Zatoński (iD) \\ 'Department of Otolaryngology, Head \\ and Neck Surgery, Wroclaw Medical \\ University, Wroclaw, Poland; \\ ${ }^{2}$ Department of Vascular Surgery, \\ Wroclaw Medical University, Wroclaw, \\ Poland
}

\begin{abstract}
Acute respiratory syndrome, associated with coronavirus 2 (SARS-CoV-2), is the most important medical and epidemic problem of today. The biggest challenge is to find an effective treatment and to reduce the need for hospitalisation. In the article, the patients with mild to moderate coronavirus disease 2019 (COVID-19) treated with doxycycline with significant improvement have been discussed. Doxycycline is a known antibiotic, but also an anti-inflammatory and immunomodulatory drug, so it seems to be ideal for the treatment of COVID-19. Doxycycline, as an easily available and low-cost medication, should be considered as a COVID-19 therapy in all patients in the first days of the symptoms of a SARS-CoV-2 infection. Due to its immunomodulatory, anti-inflammatory, cardioprotective and antiviral effects, it seems to be an ideal drug for patients with mild, moderate and severe disease. A large multicentre study is needed to evaluate the effects of this medication.
\end{abstract}

Keywords: COVID-19, COVID-19 therapy, doxycycline, SARS-CoV-2

\section{Introduction}

Acute respiratory syndrome is associated with coronavirus 2 (SARS-CoV-2), a virus which caused an unbelievable crisis around the world. As of the $23 \mathrm{rd}$ of May, 2021, 167,070,799 cases were diagnosed, 148,011,966 people have recovered, and 3,469,436 have died. ${ }^{1}$ The crisis has affected medical services and public health, but also the global economy. ${ }^{2}$ The pathophysiology of the disease is based on an infection of the upper and lower respiratory tract caused by the virus, as well as an excessive response of the immune system, or autoimmunity. ${ }^{3}$ Patients with a positive test result are recommended to stay isolated, rest and be treated with fluid therapy. ${ }^{1}$ Hospitalisation is indicated in moderate and severe cases with risk factors such as chronic lung diseases, cardiovascular diseases, obesity and diabetes. ${ }^{1}$ The immaturity of the immune system in children can protect them from the severe course of this disease and any possible complications. The infection can lead to severe lung damage, acute respiratory distress syndrome (ARDS) and, consequently, multiple organ failure. ${ }^{4}$ There are still no validated and effective guidelines for the treatment of SARS-CoV-2.

Doxycycline is a long-acting, broad-spectrum, semi-synthetic tetracycline antibiotic. It is a bacteriostatic antibiotic and blocks protein biosynthesis. A strong antiinflammatory effect of this drug has been demonstrated before. ${ }^{5,6}$ It is recommended in the guidelines for chronic sinusitis as an immunomodulating and disease-stabilising medication. ${ }^{7}$ It is also an antiviral antibiotic against RNA viruses. It is used, for example, in dengue fever. ${ }^{5}$ It has been well proven to block a cytokine storm. Malek
Correspondence: Karolina Dorobisz Email dorobiszkarolina@gmail.com 
et $\mathrm{al}^{8,10}$ in their publications highlighted the important role of doxycycline in the treatment of COVID-19.

\section{Materials and Methods}

In the paper, four cases of patients with confirmed SARS$\mathrm{CoV}-2$ infection have been presented.

\section{Case I}

A 34-year-old male, never smoked, body mass index (BMI) of 24.7, regularly practising sports. Suffering from allergic multimorbidity, and asthma controlled from early childhood. On the 21st of October 2020 he noticed a slight sore throat and headache. The next day, his symptoms including weakness, particularly sweating of the face and back, temperature of 37.3 degree Celsius, fatigue, sore throat, and occasional dry cough. A real-time polymerase chain reaction (RT-PCR) test was performed confirming SARS-CoV-2 infection. Oxygen saturation was normal, 96-98\%. Chest X-ray confirmed inflammatory lesions of the interstitial type. Blood tests showed leucocytosis and CRP level of 40. Doxycycline therapy was started initially with $200 \mathrm{mg}$, and then continued with $100 \mathrm{mg}$ for 7 days. Complete remission of symptoms was achieved after 2 doses.

\section{Case 2}

A 34-year-old female, never smoked, healthy, BMI of 22.8. Comorbidity included allergy, controlled asthma and IgA deficiency. Due to the infection of the household member, the patient was tested on the 23rd of October 2020 with a positive result. On the 25th of October 2020 the first symptoms, including a severe headache in the area of the forehead and eyes, a slight sore throat, weakness, sweating, temperature of 35.2 degree Celsius, nasal congestion without discharge, loss of smell and taste, and lack of appetite appeared. The next day, she experienced a dry cough, chest pain, feeling of arrhythmia, periodic tachycardia, heart rate of 100-120, feeling of shortness of breath, and oxygen saturation of about $90 \%$. Chest X-ray confirmed lung inflammation. Doxycycline therapy at a dose of $200 \mathrm{mg}$ was started and continued for 5 days; then, $100 \mathrm{mg}$ were administered for 7 days, after 3 doses of the drug, significant improvement was achieved, oxygen saturation returned to normal, periodically productive cough, chest pain subsided, heart rate $80-90$.

\section{Case 3}

An 83-year-old male patient with arterial hypertension, chronic obstructive pulmonary disease, diabetes and hyperlipidaemia, BMI of 28 . On the 13th of October 2020 an infection with symptoms and a fever up to 38 degree Celsius appeared. Tachypnoea. After 2 days, a test that confirmed the SARS-CoV-2 infection was performed. At the beginning of the infection (on the 13th of October 2020), treatment with doxycycline $2 \times 100 \mathrm{mg}$ was started. After three doses of the antibiotic, the patient's condition improved significantly. The patient did not require hospitalisation. Symptoms resolved after 5 days of treatment. Laboratory tests showed slight leucocytosis. Chest X-ray unchanged.

\section{Case 4}

A 59-year-old male patient, generally healthy, BMI of 25 , a physician. He suffered from symptoms of extreme weakness, pain in muscles and joints, shortness of breath and chest pain. Electrocardiography performed in the patient confirmed arrhythmia, and inflammatory markers were elevated. Chest X-ray confirmed lung lesions of the interstitial type. The patient was tested for SARS-CoV-2. On the 5 th of October 2020, the patient had a positive test result. From that day on, the therapy included doxycycline at a dose of $2 \times 100 \mathrm{mg}$. After the second dose of the antibiotic, shortness of breath and chest pain completely subsided. Pain in muscles and joints disappeared after 4 days. Treatment with antibiotics was completed after 10 days and the patient did not require hospitalisation.

\section{Discussion}

COVID-19 is an infection of the upper and lower respiratory tract. The main symptoms include fever, cough, tachypnoea and hypoxia, as well as smell and taste disorders. ${ }^{9}$ The four described cases indicate a significant influence of doxycycline on the course of the coronavirus infection in mild and moderate stages in young patients. It is difficult to unequivocally confirm the effectiveness of the medication in the presented cases, but similar observations were made by other researchers. ${ }^{3,9-12}$ In the study of Mohammud M. Alam et al, ${ }^{9}$ a description of a series of 89 elderly patients with confirmed moderate to severe COVID-19 with concomitant diseases was presented. All patients in this group were treated with doxycycline for 7 days at a dose of $100 \mathrm{mg}$ daily. The mean age of the patients was 78 years. The authors documented recovery 
in $85 \%$ of patients. Similarly, Paul A. Yates et $\mathrm{al}^{3}$ described a series of 4 cases of patients treated for COVID-19 with concomitant lung diseases, all patients improved after treatment with doxycycline. One patient in this group switched to doxycycline, despite the initial failure of azithromycin, which resulted in improvement. The described patients suffered from lung cancer or sarcoidosis. Malek et $\mathrm{al}^{10}$ also mention the possible role of doxycycline in COVID-19 therapy in combination with hydroxychloroquine instead of azithromycin. Administration of hydroxychloroquine with azithromycin causes viral remission in $100 \%$ of patients on the 5 th day of therapy. ${ }^{13}$ Treatment with azithromycin is associated with cardiotoxicity for the elderly; therefore, Malek et al. ${ }^{10}$ suggest switching to doxycycline primarily in elderly patients. Yasemin Cag et al, ${ }^{11}$ also draw attention to the success of the use of doxycycline in the therapy of dengue haemorrhagic fever, while negating the effectiveness of lopinavir in the treatment of COVID-19 patients.

Hayder M. Al-kuraishy et al ${ }^{12}$ indicate tetracyclines as a possible therapy for COVID-19 patients. They emphasise the effectiveness of doxycycline and minocycline in treatment, as well as the synergistic effect with chloroquine. Tetracyclines are effective especially in pneumonia and complications such as acute lung injury (ALI) and ARDS.

Bonzano et $\mathrm{al}^{14}$ confirmed that doxycycline at a dose of $200 \mathrm{mg}$ daily in patients with COVID-19 administered for 8 days improved the sense of smell. This improvement was associated with the modulation of ACE2 and CD147 expression in the olfactory epithelium.

Mostafa $^{15}$ confirmed that doxycycline is effective in reducing ARDS induced by COVID-19 and cytokine storms. This is related to the easy penetration of tetracyclines into the pulmonary alveoli due to lipophilicity.

Mosquera-Sulbaran and Hernandez-Fonseca ${ }^{16}$ found that doxycycline reduces virus replication by inhibiting its penetration into cultured cells.

COVID-19 induces the proliferation of mast cells in the airway mucosa, ${ }^{17}$ activates the NF-kB pathway, ${ }^{18}$ thus increasing the inflammatory response. COVID-19 is also associated with the activation of IL-1b, TNF and IL-6. Myocardial injury, as well as an increase in troponin levels, have also been documented. ${ }^{19}$ Doxycycline reduces DPP4 expression by blocking the NF-kB pathway, and consequently blocking the penetration of the virus into cells. $^{5,20}$ Doxycycline blocks IL-6, MMPs, and especially MMP-9. MMP-9 is necessary for the penetration of the virus into the cell. ${ }^{5}$ IL-6 and MMPs are cytokine storm regulators often associated with severe viral pneumonia. ${ }^{6,21}$ In vivo inhibition of CD14 +/EMMPRIN by doxycycline was found, which may inhibit penetration of SARS-CoV-2 into T cells. ${ }^{22}$

Doxycycline also has a cardioprotective effect. It regulates the MMP-2 pathway, protecting the systolic function of the heart. ${ }^{23}$ As a result, it inhibits arrhythmias caused by myocardial ischemia.

The antiviral activity of tetracyclines may be associated with increased transcription of intracellular zincfinger antiviral protein (ZAP). ${ }^{24}$ ZAP may also bind to specific target viral mRNAs and inhibit RNA translation. $^{25}$ In experimental studies, tetracycline was used to induce overexpression of the host ZAP in HEK293, rat and monkey (Vero cells) cell lines, which contributed to the inhibition of RNA viruses such as dengue virus, Ebola virus, human immunodeficiency virus, Zika virus and influenza A virus. ${ }^{24,26,27}$

\section{Conclusions}

Doxycycline, as an easily available and low-cost medication, should be considered as a COVID-19 therapy in all patients in the first days of symptoms of SARS-CoV-2 infection.

Due to its immunomodulatory, anti-inflammatory, cardioprotective and antiviral effects, it seems to be an ideal drug for patients with mild, moderate and severe type of COVID-19.

A large multicentre study to evaluate the effects of this medication is needed.

\section{Disclosure}

The authors report no conflicts of interest in this work.

\section{References}

1. Worldometers.Available from: https://www.worldometers.info/corona virus/. Accessed September 7, 2021.

2. Centers for Disease Control and Prevention. Coronavirus disease 2019 (COVID-19) treatment guidelines. Available from: https://www.cdc. gov/coronavirus/2019-ncov/index.html. Accessed September 17, 2021.

3. Yates PA, Newman SA, Oshry LJ, Glassman RH, Leone AM, Reichel E. Doxycycline treatment of high-risk COVID-19-positive patients with comorbid pulmonary disease. Ther Adv Respir Dis. 2020;14:1-5. doi:10.1177/1753466620951053

4. Shi Y, Wang Y, Shao C. COVID-19 infection: the perspectives on immune responses. Cell Death Differ. 2020;27(5):1451-1454. doi:10.1038/s41418-020-0530-3

5. Griffin MO, Fricovsky E, Ceballos G, et al. Tetracyclines: a pleiotropic family of compounds with promising therapeutic properties. Review of the literature. Am J Physiol Cell Physiol. 2010;299:C539-C548. 
6. Conforti C, Giuffrida R, Zalaudek I, et al. Doxycycline, a widely used antibiotic in dermatology with a possible anti-inflammatory action against IL-6 in COVID-19 outbreak. Dermatol Ther. 2020;33 (4): 13437.

7. Fokkens WJ, Lund VJ, Mullol J, et al. EPOS 2012: European position paper on rhinosinusitis and nasal polyps 2012. A summary for otorhinolaryngologists. Rhinology. 2012;50(1):1-12. doi:10.4193/ Rhino50E2

8. Malek AE, Granwehr BP, Kontoviannis DP. Doxycycline as a potential partner of COVID-19 therapies. 2020;21:e00864.

9. Alam MM, Mahmud S, Rahman MM, Simpson J, Aggarwal S, Ahmed Z. Clinical outcomes of early treatment with doxycycline for 89 high-risk COVID-19 patients in long-term care facilities in New York. Cureus. 2020;12(8):e9658.

10. Malek AE, Granwehr BP. Doxycycline as an alternative to azithromycin in elderly patients. Int $J$ Antimicrob Agents. 2021;57 (1):106168. doi:10.1016/j.ijantimicag.2020.106168

11. Cag Y, Icten S, Isik-Goren B, et al. A novel approach to managing COVID-19 patients; results of lopinavir plus doxycycline cohort. Eur $J$ Clin Microbiol Infect Dis. 2021;40:407-411. doi:10.1007/s10096020-04016-1

12. Al-kuraishy HM, Al-Gareeb AI, Alqarni M, Cruz-Martins N, ElSaber Batiha G. Pleiotropic effects of tetracyclines in the management of COVID-19: emerging perspectives. Front Pharmacol. 2021;12:642822. doi:10.3389/fphar.2021.642822

13. Gautret P, Lagier JC, Parola P, et al. Hydroxychloroquine and azithromycin as a treatment of COVID-19: results of an open-label non-randomized clinical trial. Int J Antimicrob Agents. 2020;56 (1):105949. doi:10.1016/j.ijantimicag.2020.105949

14. Bonzano C, Borroni D, Lancia A, Bonzano E. Doxycycline: from ocular rosacea to COVID-19 anosmia. new insight into the coronavirus outbreak. Front Med (Lausanne). 2020;7:200. doi:10.3389/ fmed.2020.00200

15. Mostafa MA. Doxycycline and minocycline drugs as a treatment proposal for inhibition of ARDS and inflammatory cytokine mediators caused by COVID19; 2020. AIJR Preprints.

16. Mosquera-Sulbaran JA, Hernández-Fonseca $H$. Tetracycline and viruses: a possible treatment for COVID-19? Arch Virol. 2021;166:1-7. doi:10.1007/s00705-020-04860-8
17. Sodhi M, Etminan M. Therapeutic potential for tetracycline in the treatment of COVID-19. Pharmacotherapy. 2020;40:487-488. doi:10.1002/phar.2395

18. Kumar N, Xin ZT, Liang Y, Ly H, Liang Y. NF-kappaB signaling differentially regulates influenza virus RNA synthesis. $J$ Virol. 2008;92:9880-9889. doi:10.1128/JVI.00909-08

19. Shi S, Qin M, Shen B, et al. Association of cardiac injury with mortality in hospitalized patients with COVID-19 in Wuhan, China. JAMA Cardiol. 2020;5:802-810. doi:10.1001/jamacardio.2020.0950

20. Choi B, Lee S, Kim SM, et al. Dipeptidyl peptidase-4 induces aortic valve calcification by inhibiting insulin-like growth factor-1 signaling in valvular interstitial cells. Circulation. 2017;16:1935-1950. doi:10.1161/CIRCULATIONAHA.116.024270

21. Kong MYF, Whitley RJ, Peng N, et al. Matrix metalloproteinase-9 mediates RSV infection in vitro and in vivo. Viruses. 2015;7:4230-4253. doi:10.3390/v7082817

22. Emingil G, Atilla G, Sorsa T, et al. The effect of adjunctive subantimicrobial dose doxycycline therapy on GCF EMMPRIN levels in chronic periodontitis. J Periodontol. 2008;79:469-476. doi:10.1902/ jop.2008.070165

23. Villarreal FJ, Griffin M, Omens J, Dillmann W, Nguyen J, Covell J. Early short-term treatment with doxycycline modulates postinfarction left ventricular remodeling. J Circulation. 2003;108:1487-1492. doi:10.1161/01.CIR.0000089090.05757.34

24. Tang Q, Wang X, Gao G. The short form of the zinc finger antiviral protein inhibits Influenza A virus protein expression and is antagonized by the virus-encoded NS1. J Virol. 2017;91:e01909-e01916. doi:10.1128/JVI.01909-16

25. Guo X, Carroll J-WN, MacDonald MR, Goff SP, Gao G. The zinc finger antiviral protein directly binds to specific viral mRNAs through the $\mathrm{CCCH}$ zinc finger motifs. $J$ Virol. 2004;78:12781-12787. doi:10.1128/JVI.78.23.12781-12787.2004

26. Bick MJ, Carroll J-WN, Gao G, Goff SP, Rice CM, MacDonald MR. Expression of the zinc-finger antiviral protein inhibits alphavirus replication. $J$ Virol. 2003;77:11555-11562. doi:10.1128/ JVI.77.21.11555-11562.2003

27. Li MMH, Aguilar EG, Michailidis E. Characterization of novel splice variants of zinc finger antiviral protein (ZAP). J Virol. 2019;93: e00715-e00719.
Therapeutics and Clinical Risk Management

\section{Publish your work in this journal}

Therapeutics and Clinical Risk Management is an international, peerreviewed journal of clinical therapeutics and risk management, focusing on concise rapid reporting of clinical studies in all therapeutic areas, outcomes, safety, and programs for the effective, safe, and sustained use of medicines. This journal is indexed on PubMed Central, CAS,

\section{Dovepress}

EMBase, Scopus and the Elsevier Bibliographic databases. The manuscript management system is completely online and includes a very quick and fair peer-review system, which is all easy to use. Visit http://www.dovepress.com/testimonials.php to read real quotes from published authors. 